\title{
Applications of The Penalty Function Method in Constrained Optimal Control Problems *
}

\author{
An-qing Xing \\ Department of Mathematics and Statistics \\ University of Regina \\ Regina, Saskatchewan \\ Canada S4S OA2
}

\begin{abstract}
This paper uses the penalty function method to solve constrained optimal control problems. Under suitable assumptions, we can solve a constrained optimal control problem by solving a sequence of unconstrained optimal control problems. In turn, the constrained solution to the main problem can be obtained as the limit of the solutions of the sequence. In using the penalty function method to solve constrained optimal control problems, it is usually assumed that each of the modified unconstrained optimal control problems has at least one solution. Here we establish an existence theorem for those problems. Two numerical examples are presented to demonstrate the findings.
\end{abstract}

Key words: Optimal control, inequality constraints, penalty function AMS classification index: 49B36

\section{Introduction}

It is well known that the Lagrange multiplier method is a technique for transforming a problem of minimizing a real valued function subject to some equality constraints into an unconstrained problem. The penalty function method which can be considered as a generalization of the Lagrange multiplier method, is based on transforming a constrained optimization problem into a single or a sequence of unconstrained optimization problems. The initial suggestions for studying constrained optimization problems via unconstrained

* Received: April, 1989; Revised September, 1989 
problems were given by Courant ( $c f$. [3] ). In recent years, much research effort has been devoted to implementing the penalty function method to solve constrained optimal control problems ( $c f .[2,5,6,7]$ ). The first penalty function approach to optimal control problems was probably done by Russell (cf. [7] ). This paper generalizes the results obtained in [6] and [7]. Section 2 uses the penalty function method to solve constrained optimal control problems and proves a convergence theorem. Section 2 also presents an existence theorem for the modified optimal control problems. Two numerical examples are presented in Section 3 to show the applicability of the penalty function method.

\section{Theoretical Results}

Consider the following constrained optimal control problem:

$$
\left.\min J(u)=\min \left[\int_{t_{0}}^{t_{1}} f d x(t), u(t), t\right) d t\right]
$$

subject to

$$
\dot{x}=f(x(t), u(t), t), x\left(t_{0}\right)=x_{I}, x\left(t_{1}\right)=x_{T},
$$

and the mixed inequality constraints

$$
g_{i}(x(t), u(t), t) \geqslant 0, \quad i=1, \ldots, m
$$

where

$$
\begin{gathered}
x=\left(x_{1}, \ldots, x_{n}\right)^{r} \in R^{n}, u=\left(u_{1}, \ldots, u_{r}\right)^{r} \in U \subset R^{r}, \\
f=\left(f_{1}, \ldots, f_{n}\right)^{r} \in R^{n},
\end{gathered}
$$

and $f_{i}(i=0, \ldots, n)$ and $g_{i}(i=1, \ldots, m)$ are all continuous functions on $R^{n+r+1}$. $x$ and $u$ are the state and the control of the system respectively.

By using the penalty function method we may consider the following unconstrained optimal control problem instead:

$$
\min P(u, s)=\min \left[J(u)+\frac{s}{2} \sum_{i=1}^{m} \int_{i_{0}}^{t_{1}}\left(g_{i}(x(t), u(t), t)\right)^{2} H\left(g_{i}\right) d t\right]
$$

subject to (2.2), where $H(g)=0$ if $g \geqslant 0$ and 1 if $g<0$, and $s$ is the penalty factor. This unconstrained problem can now be solved by either the 
maximum principle or by the dynamic programming technique. Suppose, for each $s$, we can solve the unconstrained optimal control problem (2.4) subject to (2.2) and obtain the optimal control $u^{s}(t)$ and the minimum performance measure $P^{s}$. Then it can be proved that

$$
\lim _{s \rightarrow \infty} p^{s}=J^{*}
$$

where $J_{*}$ is the minimum performance measure for the original problem (2.1) - (2.3) ( $\mathrm{cf}$. [6] ). It can also be proved, under certain conditions, that the sequence of the solutions $u^{s}(t)$ converges weakly to an optimal solution of the original problem (2.1) - (2.3). To see this, let us consider the following constrained optimal control problem:

$$
\min J(u)=\min \int_{i_{0}}^{t_{1}}\left[f f_{0}(x, t)+C(t) u(t)\right] d t
$$

subject to

$$
\begin{gathered}
\dot{x}=f(x, t)+B(t) u(t), \\
x\left(t_{0}\right)=x_{1}, \quad x\left(t_{1}\right)=x_{T}, \\
g_{i}(x, t) \geqslant 0, \quad i=1, \cdots, m, \\
u(t) \in U \subset R^{r}
\end{gathered}
$$

where $g_{i}$ are continuous functions on $R^{n+1}$,

$$
C(t)=\left(c_{1}(t), \cdots, c_{r}(t)\right) \in R^{r},
$$

and $B(t)$ is an $n \times r$ matrix. Assume that each $u_{i}(t) \in L^{2}\left[t_{0}, t_{1}\right](i=1, \ldots, r)$, $U$ is a compact and convex set in $R^{r}$, and $t_{0}, t_{1}\left(t_{0}<t_{1}\right)$ are fixed, where $L^{2}\left[t_{0}, t_{1}\right]$ is the Hilbert space of all measurable functions which are square integrable. We also assume that $f(x, t)$ is continuous and bounded on $R^{n} \times\left[t_{0}, t_{1}\right]$ and $B(t)$ is continuous on $\left[t_{0}, t_{1}\right]$. A control $u(t) \in L^{2}\left[t_{0}, t_{1}\right]$ is said to be admissible if $u(t) \in U$ and its response $x(t)$ satisfies the constraints (2.7) and (2.8). Suppose that the constrained optimal control problem (2.5) - (2.8) has at least one optimal solution $u^{*}(t)$. Using the penalty function method we consider the following unconstrained optimal control problem:

$$
\min P(u)=\min \left[J(u)+v_{k} \sum_{i=1}^{m} \int_{i_{0}}^{t_{1}}\left(g_{i}(x, t)\right)^{2} H\left(g_{i}\right) d t\right]
$$


subject to (2.6) - (2.7) and $u(t) \in U$, where $\left\{v_{k}\right\}$ is a sequence with $\lim _{k \rightarrow \infty} v_{k}=\infty$.

Now, we have

Theorem 2.1: If, for every $v_{k}>0$, there exists an optimal solution $u^{k}(t)$ for the unconstrained optimal control problem (2.9), then there exists a subsequence of $\left\{u^{k}(t)\right\}$ which converges weakly to an optimal solution of the constrained optimal control problem (2.5) - (2.8).

Proof: Let $u^{k}(t)=\left(u_{1}^{k}(t), \cdots, u_{r}^{k}(t)\right)$. Then each function $u_{i}^{k}(t)$ belongs to $L^{2}\left[t_{0}, t_{1}\right]$ Since $u^{k}(t) \in U$ and $U$ is bounded, the functions $u^{k_{t}}(t)$ $(k=1,2, \cdots)$, for every fixed $i(i=1, \ldots, r)$, all belong to some ball in $L^{2}\left[t_{0}, t_{1}\right]$. Since $L^{2}\left[t_{0}, t_{1}\right]$ is a reflexive space, the sequence $u_{i}^{k}(t)(k=1,2, \cdots)$ has a weakly convergent subsequence ( $c f .[8]$ ). We shall simply assume that the sequence

$$
u_{i}{ }^{2}(t), u_{i}^{2}(t), \ldots, u_{i}^{k}(t), \cdots
$$

itself converges weakly to some function $\bar{u}_{i}(t)$ for $i=1, \ldots, r$.

Let us now prove that

$$
\bar{u}(t) \stackrel{\Delta}{=}\left(\bar{u}_{1}(t), \ldots, \bar{u}_{r}(t)\right)
$$

is in $U$ for almost all $\mathrm{t}$ in $\left[t_{0}, t_{1}\right]$. Since $U$ is a compact and convex set in $R^{r}$, it is the intersection of a finite or countable number of closed half-spaces (cf. [1]). That is, $U$ is the set of points $u=\left(u_{1}, \ldots, u_{r}\right)$ in $R^{r}$ which satisfy a system of countable linear inequalities:

$$
\sum_{i=1}^{r} \alpha_{i}{ }^{\prime} u_{i} \leqslant \beta^{\prime} \quad j=1,2, \cdots
$$

Note that if $U$ is the intersection of a finite number of closed half-spaces, then $U$ is simply a polyhedron. For a fixed $j$, let $L(u)=\sum_{i=1}^{r} \alpha_{i}^{j} u_{i}$ and let $m_{j}$ be the set of all points $t \in\left[t_{0}, t_{1}\right]$ for which $L(\bar{u}(t))>\beta^{\prime}$ and let $w(t)$ be the characteristic function of $m_{j}$. Then $w(t)$ is bounded and measurable. So we have

$$
w(t) \in L^{2}\left[t_{0}, t_{1}\right]
$$

and

$$
\lim _{k \rightarrow \infty} \int_{t_{0}}^{t_{1}} w(t)\left(L(\bar{u}(t))-L\left(u^{k}(t)\right)\right] d t=0
$$


since $u^{k}(t)$ converges weakly to $\bar{u}(t)$. That is,

$$
\lim _{k \rightarrow \infty} \int_{m_{j}}\left[L(\bar{u}(t))-L\left(u^{k}(t)\right)\right] d t=0 .
$$

Since $u^{k}(t) \in U$, we have

$$
L\left(u^{k}(t)\right) \leqslant \beta^{\prime}
$$

and

$$
L(\bar{u}(t))-L\left(u^{k}(t)\right)>0
$$

for each $t \in m_{j}$. Therefore, by (2.11), the measure of $m_{J}$ is zero, which means that

$$
\sum_{i=1}^{r} \alpha_{i} \bar{u}_{i}(t) \leqslant \beta^{j}
$$

for almost all $t \in\left[t_{0}, t_{1}\right]$. Since $j$ is arbitrary, inequality (2.12) holds for $j=1,2, \cdots$ for almost all $t \in\left[t_{0}, t_{1}\right]$. That is, for almost all $t \in\left[t_{0}, t_{1}\right]$, we have $\bar{u}(t) \in U$.

Changing the value of $\bar{u}(t)$ on a set with measure zero does not disturb the weak convergence of the sequence $(2.10)$ to the function $\bar{u}_{i}(i=1, \ldots, r)$. Thus we may assume that $\bar{u}(t) \in U$ for all $t \in\left[t_{0}, t_{1}\right]$.

Let $x^{k}(t)$ denote the response corresponding to $u^{k}(t)$. That is

$$
\begin{gathered}
x^{k}(t)=x_{I}+\int_{t_{0}}^{t}\left[f\left(x^{k}(s), s\right)+B(s) u^{k}(s)\right] d s, \\
x^{k}\left(t_{1}\right)=x_{T} .
\end{gathered}
$$

Since $f($.$) is bounded and continuous, \left\{x^{k}(t)\right\}$ is equicontinuous and bounded uniformly. Therefore, $\left\{x^{k}(t)\right\}$ contains a uniformly convergent subsequence (cf. [4] ). For simplicity, we can assume that $\left\{x^{k}(t)\right\}$ itself converges to some function, say, $\bar{x}(t)$.

Passing to the limit, it follows from (2.13) and (2.14) that

$$
\begin{gathered}
\bar{x}(t)=x_{I}+\int_{t_{0}}^{t}[f(\bar{x}(s), s)+B(s) \bar{u}(s)] d s, \\
\bar{x}\left(t_{1}\right)=x_{T} .
\end{gathered}
$$


Now, we prove that $g_{i}(\bar{x}(t), t) \geqslant 0$ for each $i=1, \ldots, m$ and for all $t \in\left[t_{0}, t_{1}\right]$. If not, then, for some $i$, there is a $K$, a subinterval $\left[t_{3}, t_{4}\right] \subset\left[t_{0}, t_{1}\right]$ and a positive number $\rho$ such that

$$
g_{i}\left(x^{k}(t), t\right) \leqslant-\rho
$$

for all $t \in\left[t_{3}, t_{4}\right]$ and for all $k \geqslant K$, and this would give

$$
\lim _{k \rightarrow \infty} P\left(u^{k}(t)\right)=+\infty
$$

This is a contradiction since $u^{k}(t)$ is the optimal solution to problem (2.9) and therefore

$$
P\left(u^{k}(t)\right) \leqslant P\left(u^{*}(t)\right)=J\left(u^{*}(t)\right) \stackrel{\Delta}{=} J^{*}
$$

where $u^{*}(t)$ is the optimal solution to (2.5) - (2.8) (by assumption, there exists at least one such solution). So, we have

$$
g(\bar{x}(t), t) \geqslant 0, \quad t \in\left[t_{0}, t_{1}\right]
$$

Therefore $\bar{u}(t)$ is admissible and

$$
J(\bar{u}(t))=P(\bar{u}(t))=\lim _{k \rightarrow \infty} P\left(u^{k}(t)\right) \leqslant \lim _{k \rightarrow \infty} P\left(u^{*}(t)\right)=J\left(u^{*}(t)\right)
$$

since $u^{k}(t)$ converges weakly to $\bar{u}(t)$ and $x^{k}(t)$ converges uniformly to $\bar{x}(t)$.

Since $\bar{u}(t)$ is admissible, it follows, from (2.18), that $\bar{u}(t)$ is an optimal solution to the constrained optimal control problem (2.5) - (2.8) and this completes the proof.

Since the state constraint in problem (2.5) - (2.8) is time dependent, Theorem 2.1 generalizes the result obtained in [7] in which the state constraint is assumed to be time invariant.

Many authors have used the penalty function method to solve constrained optimal control problems and different results have been obtained ( $c f .[2,6])$. But in the proofs of convergence theorems, a common assumption has been made, that is, it is assumed that the unconstrained optimal control problem (2.9) attains its minimum for each $k$. Now, we give the conditions which ensure the existence of the solution to the unconstrained optimal control problem (2.9). Here, we consider the following constrained optimal control problem 


$$
\min J(u)=\min \int_{t_{0}}^{t_{1}} f_{0}(x, u, t) d t
$$

subject to

$$
\begin{gathered}
\dot{x}=A(t) x(t)+B(t) u(t), \quad x\left(t_{0}\right)=x_{I}, x\left(t_{1}\right)=x_{T}, \\
g_{i}(x, t)=D_{i}(t) x(t) \geqslant 0, \quad i=1, \ldots, m, \\
u(t) \in U, \quad t \in\left[t_{0}, t_{1}\right],
\end{gathered}
$$

where $A(t)$ is an $n$ by $n$ matrix, $B(t)$ is an $n$ by $r$ matrix, and each $D_{i}(t) \in R^{n}$ $(i=1, \ldots, m)$. Then we have

Theorem 2.2: Assume that $f_{0}(x, u, t)$ in (2.19) is continuously differentiable and convex in $x$ and $u$. Then, for every $v_{k}$, there exists an optimal solution to the unconstrained optimal control problem (2.9) with

$$
f_{0}(x, t)+C(t) u(t), \quad f(x, t), \quad g_{i}(x, t)
$$

replaced by

$$
f_{0}(x, u, t), \quad A(t) x(t)+B(t) u(t), \quad D_{i}(t) x
$$

respectively.

Proof: Let $x(t)$ be the response corresponding to $u(t)$ and define

$$
\Omega=\left\{u(t) \mid u(t) \in U, t \in\left[t_{0}, t_{1}\right] ; x\left(t_{0}\right)=x_{I}, x\left(t_{1}\right)=x_{T}\right\} .
$$

It is obvious that $\Omega$ is a convex set. That is, for any $u^{1}, u^{2} \in \Omega$ and $q \in[0,1\}$ we have

$$
u=q u^{1}+(1-q) u^{2} \in \Omega .
$$

Furthermore, the corresponding response to $u$ is

$$
q x^{1}(t)+(1-q) x^{2}(t)
$$

where $x^{1}(t)$ and $x^{2}(t)$ are the responses corresponding to $u^{1}$ and $u^{2}$ respectively.

Now, we show that $P(u)$ in $(2.9)$ is a convex functional of $u$. Let $\psi(g)=g^{2} H(g)$. Then $P(u)$ has the form

$$
P(u)=\int_{t_{0}}^{t_{1}} f_{0}(x, u, t) d t+v_{k} \int_{t_{0}}^{t_{1}} \sum_{i=1}^{m} \psi\left(D_{i}(t) x\right) d t .
$$

Because of the convexity of $f_{0}$ and $\psi$ we have 


$$
\begin{gathered}
P\left(q u^{1}+(1-q) u^{2}\right)=\int_{t_{0}}^{t_{1}} f_{0}\left(q x^{1}+(1-q) x^{2}, q u^{1}+(1-q) u^{2}, t\right) d t \\
+v_{k} \int_{t_{0}}^{t_{i}} \sum_{i=1}^{m} \psi\left(q D_{i} x^{1}+(1-q) D_{i} x^{2}\right) d t \\
\leqslant \int_{t_{0}}^{t_{1}}\left[q f_{0}\left(x^{1}, u^{1}, t\right)+(1-q) f_{0}\left(x^{2}, u^{2}, t\right)\right] d t \\
+v_{k} \int_{t_{0}}^{t_{1}} \sum_{i=1}^{m}\left[q \psi\left(D_{i}(t) x^{1}\right)+(1-q) \psi\left(D_{i}(t) x^{2}\right)\right] d t \\
=q P\left(u^{1}\right)+(1-q) P\left(u^{2}\right) .
\end{gathered}
$$

So, $P(u)$ is convex.

From Polyak' theorem ( cf. [9] ) we know that $P(u)$ is weakly lower semicontinuous. Since $\psi(t) \geqslant 0$, it follows that

$$
\inf _{u \in \Omega} P(u) \stackrel{\Delta}{=} P^{*} \geqslant J^{*}
$$

Let $\left\{u^{k}\right\} \subset \Omega$ be such that

$$
\lim _{k \rightarrow \infty} P\left(u^{k}\right)=P^{*}
$$

It can be easily proved that the set of responses for all $u \in \Omega$ is bounded. Therefore, using the method in the proof of Theorem 2.1 we can choose a subsequence $\left\{u^{k_{1}}\right\}$ converging weakly to some $u^{*} \in \Omega$. Finally, since $P(u)$ is weakly lower semicontinuous we have

$$
P\left(u^{*}\right) \leqslant \lim _{l \rightarrow \infty} P\left(u^{k_{l}}\right)=P^{*}
$$

which means that $u^{*}$ is an optimal solution to the unconstrained optimal control problem (2.9). This completes the proof.

Theorems 2.1 and 2.2 imply that we may solve a constrained optimal control problem by solving the corresponding sequence of unconstrained optimal control problems. It should be noted that we may also transform the above formulated problems into optimal control problems with free terminal state by the penalty function method. This will be demonstrated in the next section. 


\section{Two Numerical Examples}

The key feature of the penalty function method is that it transforms a constrained optimal control problem into a sequence of unconstrained optimal control problems. As mentioned previously, these unconstrained problems can be easily solved by either the maximum principle or the dynamic programming technique. This section uses two numerical examples to show the applicability of this method. The gradient method is employed to solve an unconstrained optimal control problem.

In the following example the penalty function method is used to solve an optimal control problem with fixed terminal state.

Example 3.1: Consider the minimization of

$$
J=\int_{0}^{1} u^{2}(t) d t
$$

for the system

$$
\dot{x}=-x+u, \quad x(0)=1, \quad x(1)=0 .
$$

In order to transform the problem into a problem with free terminal state, we may consider the optimal control problem of minimizing

$$
J=s x^{2}(1)+\int_{0}^{1} u^{2}(t) d t
$$

subject to

$$
\dot{x}=-x+u, \quad x(0)=1 .
$$

This problem can now be solved by the gradient method. A Fortran program is written to find the optimal control and the optimal trajectory for the problem at hand. Numerical integrations are carried out by using the fourth-order Runge-Kutta-Gill method and the Simpson's composite rule with an integration interval of 0.1 unit and with double precision arithmetic. The iteration stops when either the change of the cost function $\left|J^{(i)}-J^{(i+1)}\right| \leqslant 10^{-6}$ or the norm of the gradient

$$
\left\|\frac{\partial H}{\partial u^{i}(t)}\right\|=\left[\int_{0}^{1}\left(\frac{\partial H}{\partial u^{i}(t)}\right)^{\tau}\left(\frac{\partial H}{\partial u^{i}(t)}\right)\right]^{\frac{1}{2}} \leqslant 10^{-2} .
$$


The initial guess of the control is $u^{0}(t)=-0.6$. To ensure that a monotonically decreasing sequence of performance indices is generated, each trial control is required to provide a smaller performance measure than the proceeding one. This is accomplished by halving the step size and regenerating any trial control that increased the performance measure.

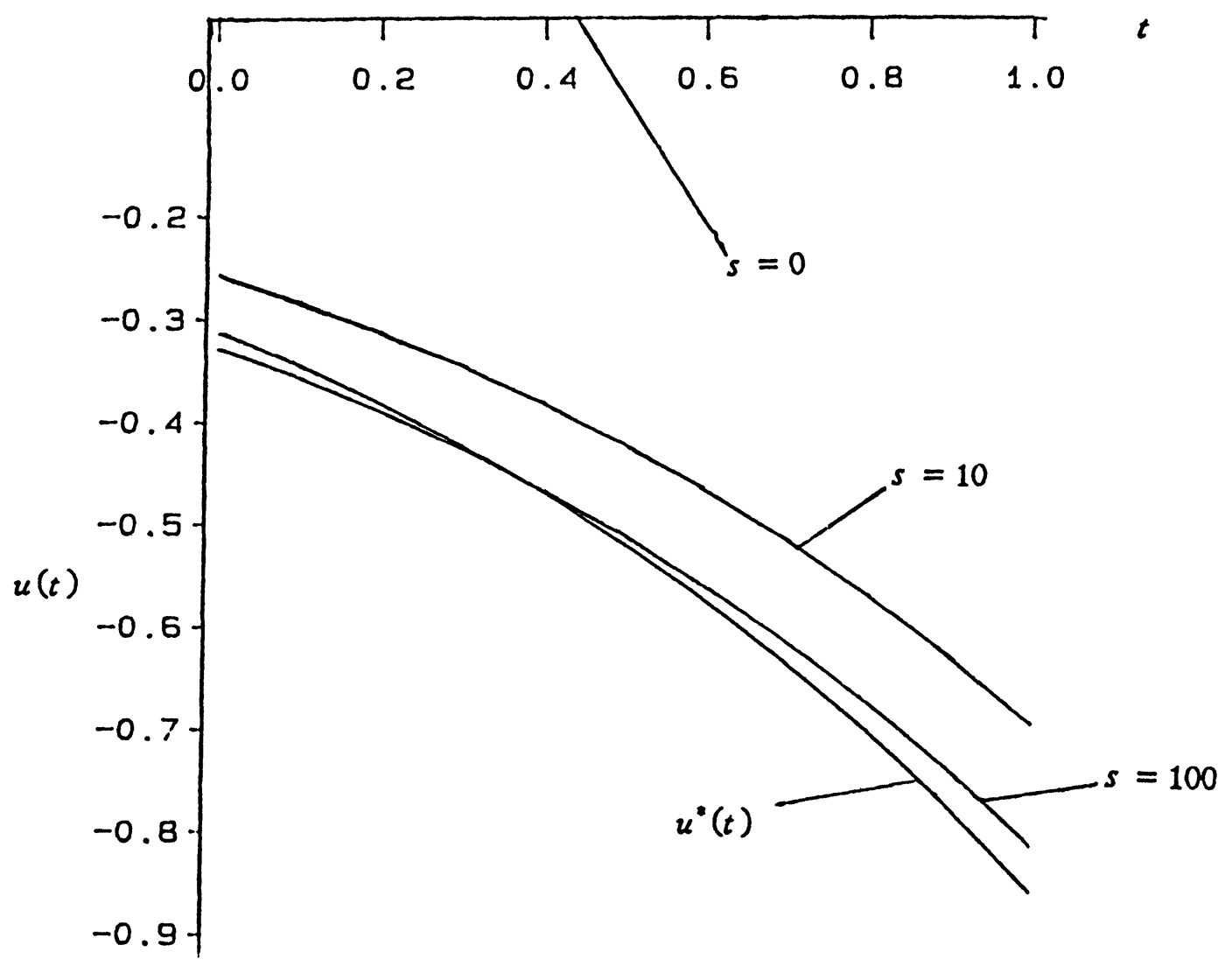

Fig. 3.1 (a): Optimal controls for different penalty factors 


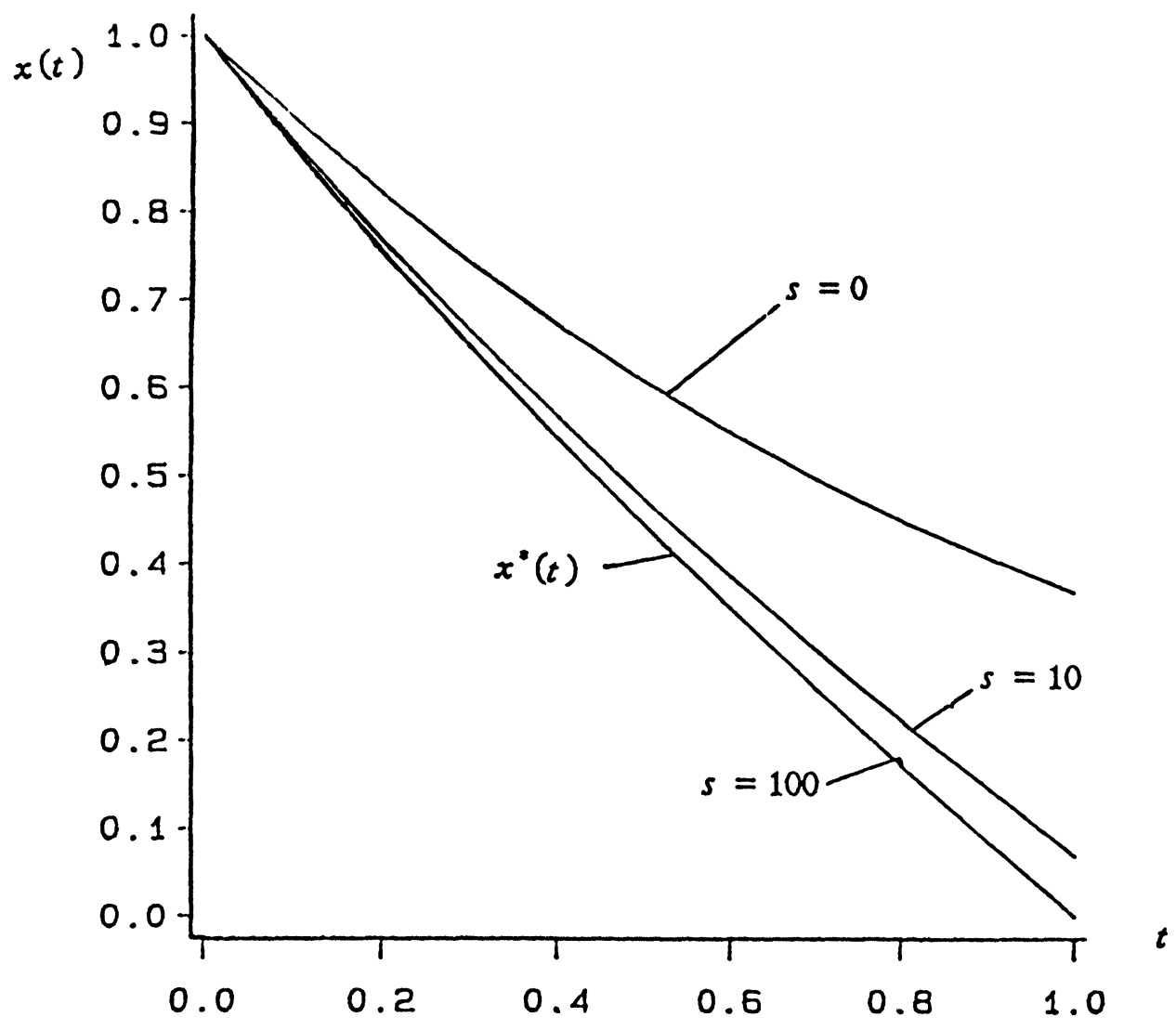

Fig. 3.1 (b): Optimal trajectories for different penalty factors

For the linear quadratic problem (3.3) - (3.4) analytical solution can be obtained as

$$
u^{s}(t)=-\frac{c}{2} e^{t}, \quad x^{s}(t)=c_{1} e^{-t}-\frac{c}{4} e^{t}
$$

where

$$
c=\frac{4}{\frac{2 e}{s}+\left(e^{2}-1\right)}, \quad c_{1}=\frac{\frac{2 e}{s}+e^{2}}{\frac{2 e}{s}+\left(e^{2}-1\right)}
$$

Let $s$ go to infinity. Then

$$
\lim _{s \rightarrow \infty} u^{s}(t)=u^{*}(t)=-\frac{2}{e^{2}-1} e^{t}, \lim _{s \rightarrow \infty} x^{s}(t)=x^{*}(t)=\frac{e^{2}}{e^{2}-1} e^{-t}-\frac{1}{e^{2}-1} e^{t}
$$

which is the optimal solution to the original problem (3.1) - (3.2). 
The numerical solutions for $s=0,10$ and 100 are compared with the analytical solutions $u^{*}(t)$ and $x^{*}(t)$ as shown in Fig. 3.1. It can be seen that the numerical solutions are forced to satisfy the terminal condition and converge to the true solutions as the penalty factor $s$ gets bigger.

Example 3.2: Consider a brachistochrone problem with an inequality constraint on the admissible state space. Specially, consider a particle falling in a constant gravitational field $g$ for a fixed time $t_{T}$ with a given initial speed $x_{3}(0)$. the purpose is to adjust the path of the particle such that final value of the horizontal coordinate $x_{1}\left(t_{T}\right)$ is maximized. The value of the vertical coordinate $x_{2}\left(t_{T}\right)$ is unspecified at the final time. This problem can be expressed by the following constrained optimal control problem:

$$
\min J=\min \left[-x_{1}\left(t_{T}\right)\right]
$$

subject to

$$
\begin{array}{ll}
\dot{x}_{1}=x_{3} \cos (u), & x_{1}(0)=0, \\
\dot{x}_{2}=x_{3} \sin (u), & x_{2}(0)=0, \\
\dot{x}_{3}=8 \sin (u), & x_{3}(0)=0.07195
\end{array}
$$

and the inequality constraint

$$
h\left(x_{1}, x_{2}, x_{3}\right)=0.2+0.4 x_{1}-x_{2} \geqslant 0
$$

where $g=1, t_{T}=1.8$.

This problem can be transformed, by the penalty function method, into the unconstrained optimal control problem of minimizing

$$
J=-x_{1}\left(t_{T}\right)+\frac{s}{2} \int_{0}^{t_{T}}\left(h\left(x_{1}, x_{2}, x_{3}\right)\right)^{2} H(h) d t
$$

subject to (3.6) - (3.8). Then the computation by the gradient method proceeds in the usual way. The Hamiltonian for this problem is

$$
H=\frac{s}{2} h^{2} H(h)+\lambda_{1} x_{3} \cos (u)+\lambda_{2} x_{3} \sin (u)+\lambda_{3} \sin (u),
$$

the adjoint system is

$$
\begin{aligned}
& \dot{\lambda}_{1}=-0.4 \operatorname{sh} H(h), \quad \lambda_{1}\left(t_{T}\right)=-1, \\
& \dot{\lambda}_{2}=\operatorname{sh} H(h), \quad \lambda_{2}\left(t_{T}\right)=0,
\end{aligned}
$$




$$
\dot{\lambda}_{3}=-\lambda_{1} \cos (u)-\lambda_{2} \sin (u), \quad \lambda_{3}\left(t_{T}\right)=0
$$

and the gradient is

$$
\frac{\partial H}{\partial u}=-\lambda_{1} x_{3} \sin (u)+\lambda_{2} x_{3} \cos (u)+\lambda_{3} \cos (u) .
$$

A Fortran program is written to solve the brachistochrone problem using the penalty function method. Again the numerical integrations are carried out using the fourth-order Runge-Kutta-Gill method and Simpson's composite rule with double precision arithmetic. The integral interval is 0.1 unit and the initial guess of the control is $u^{\alpha}(t)=\frac{\pi}{6}$.

The results show that if $s=0$, no inequality constraint is imposed on the state variables and the solution violates the constraint line $x_{2}=0.4 x_{1}+0.2$. If $s=10.0$ the solution is closer to the true solution. For $s=1000$ or greater, the solution is almost indistinguishable from the true solution which lies above the constraint line (see Fig. 3.2).

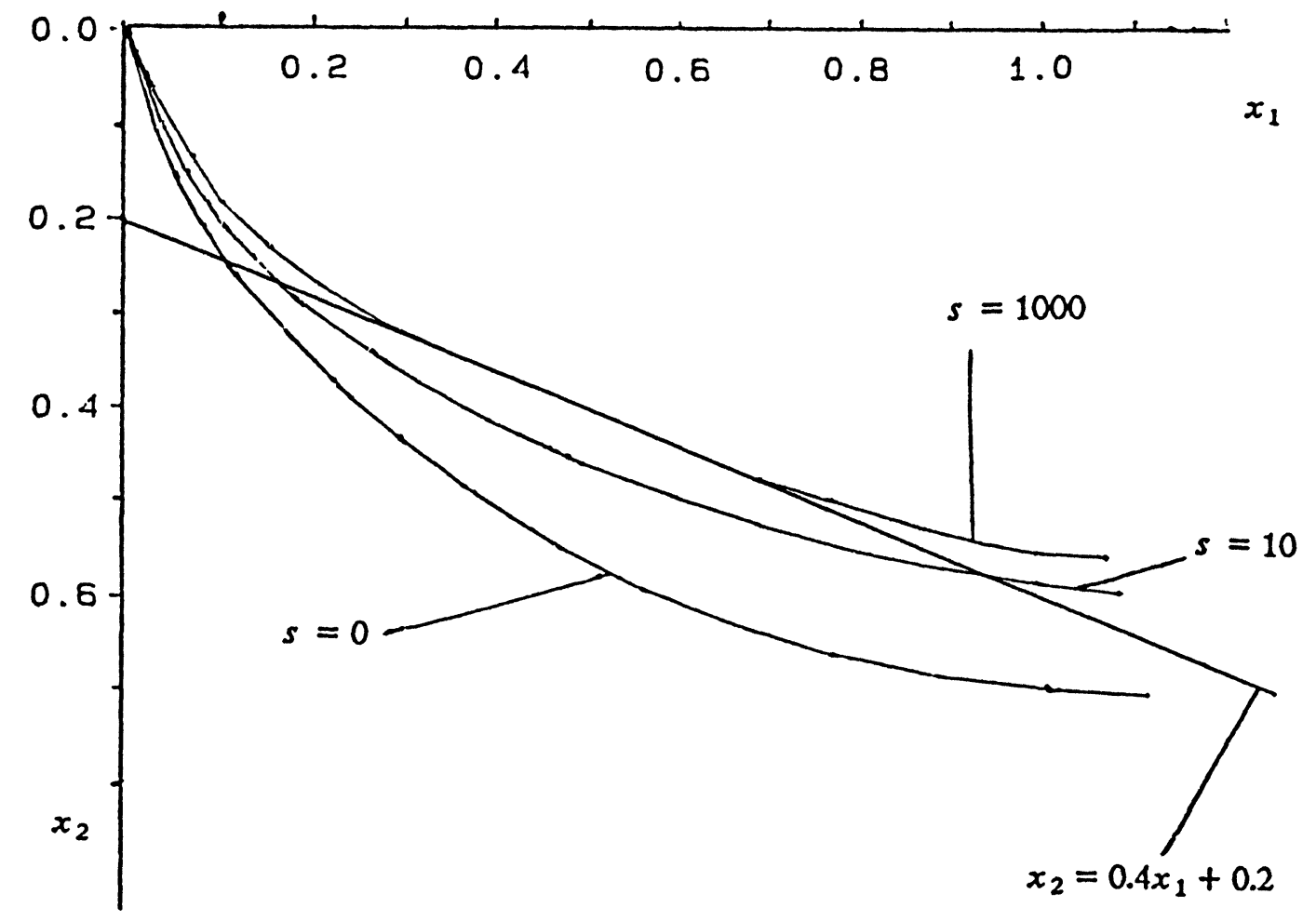

Fig. 3.2: Optimal trajectories for different penalty factors 


\section{Concluding Remarks}

This paper uses the penalty function method to solve constrained optimal control problems. It first transforms a constrained optimal control problem into a sequence of unconstrained optimal control problems. Then, by solving these unconstrained problems, a sequence of solutions is obtained. It is shown, under certain conditions, that the sequence of solutions contains a subsequence which converges to an optimal solution to the original constrained optimal control problem. This paper also presents an existence theorem for the unconstrained problems. Two numerical examples are presented to illustrate the findings. The numerical results also show that the convergence to the true solution becomes slower when the penalty factor gets bigger. This is a disadvantage of the penalty function method. However, it suggests a general method to deal with the constrained optimal control problems. Finally, if we treat (2.2) as a equality constraint, then the constrained optimal control problem can be transformed into a sequence of variational problems and parallel results can be obtained.

Acknowledgement: The author wishes to thank Professor S. K. Kaul for his helpful suggestions.

\section{References}

[1] P. Alexandroff and H. Hope, Topology, Berlin: Springer (1935).

[2] $\mathrm{Zu}$ Hao Chen, The mixed penalty function method for solving constrained optimal control problems, Control theory Appl, 1, 98-109 (1984).

[3] R. Courant, Variational method for the solution of problems of equilibrium and vibrations, Bull. Am. Math. Soc., 49, 1-23 (1943).

[4] L. M. Graves, The theory of functions of real variables, McGraw-Hill Book Company, Inc., New York (1956).

[5] D. Q. Mayne and E. Polak, An exact penalty function algorithm for optimal control problems with control and terminal equality constraints, JOT A, 32(2), 211-246 (1980).

[6] K. Okamura, Some mathematical theory of the penalty method for solving optimum control problems, J. SIAM Control, Ser. A, 2(3), 317-331 (1965). 
[7] D. L. Russell, Penalty functions and bounded phase coordinate control, J. SIAM Control, Ser. A 2(3), 409-422 (1965).

[8] Martin Schechter, Principles of functional analysis, Academic Press, Inc. New York (1971).

[9] Arthur Wouk, A course of applied functional analysis, John Wiley \& Sons Inc, New York (1979). 


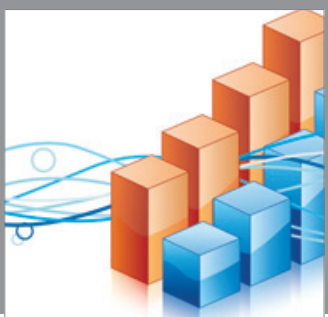

Advances in

Operations Research

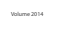

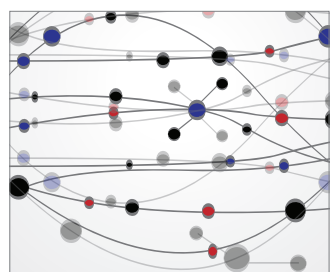

\section{The Scientific} World Journal
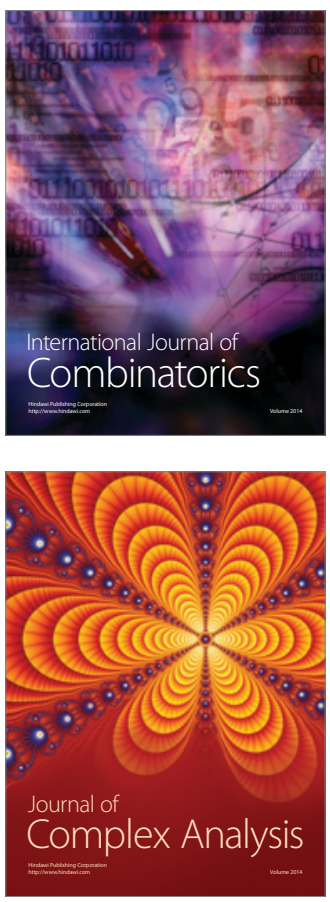

International Journal of

Mathematics and

Mathematical

Sciences
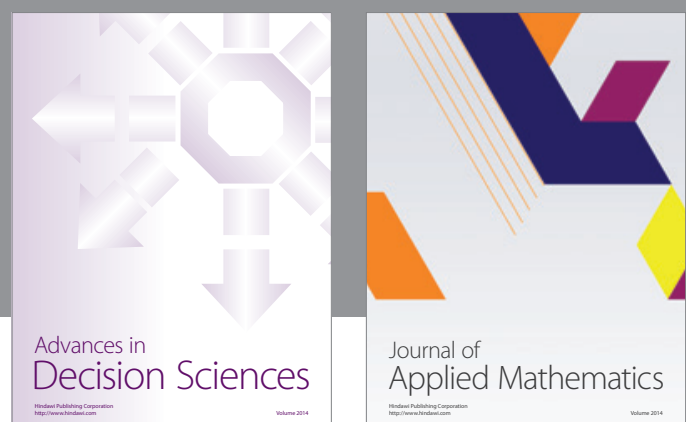

Journal of

Applied Mathematics
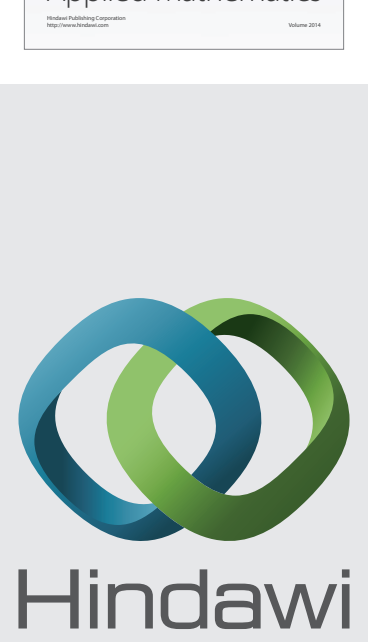

Submit your manuscripts at http://www.hindawi.com
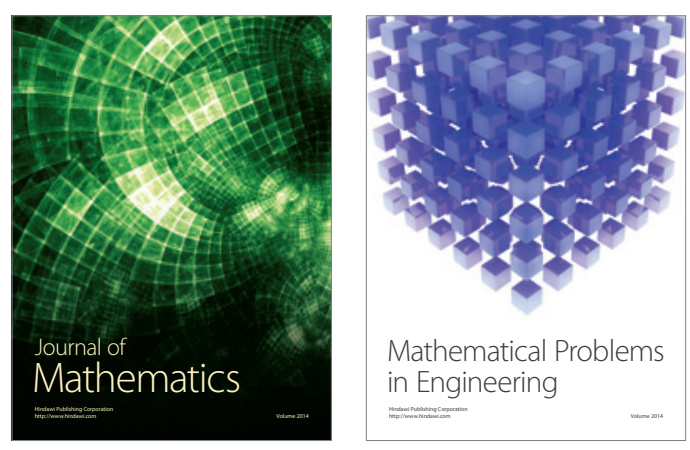

Mathematical Problems in Engineering
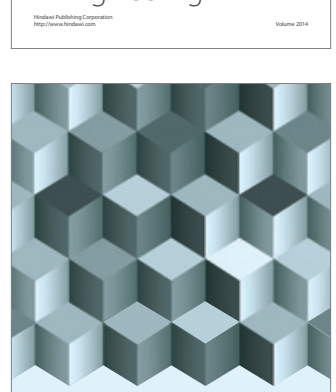

Journal of

Function Spaces
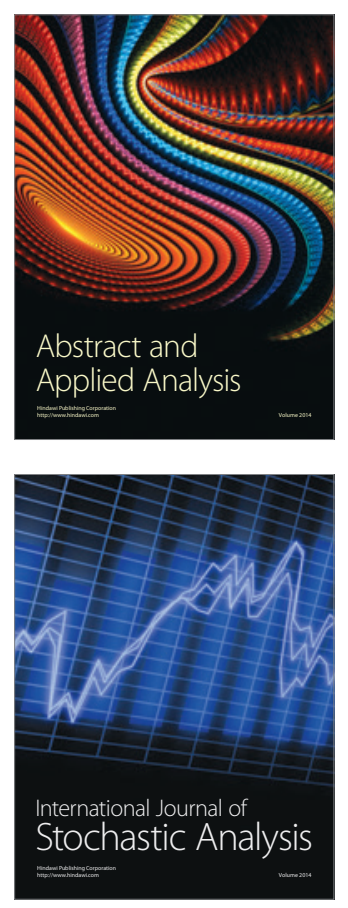

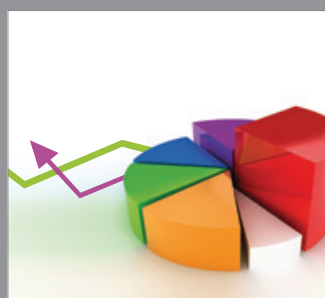

ournal of

Probability and Statistics

Promensencen
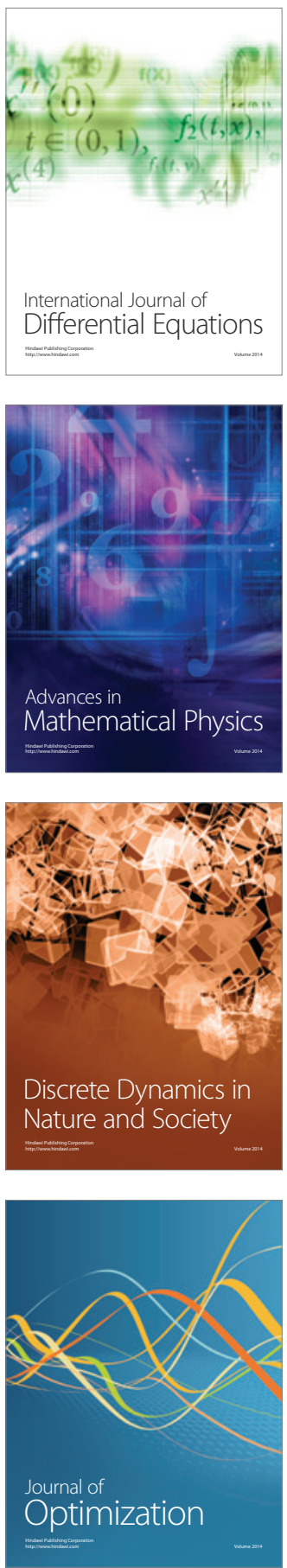